\title{
Isokinetic Peak of Torque and Fatigue Index in Simulated Wheelchair Propulsion in Elite Wheelchair Rugby Players
}

\author{
Felipe Machadoㅁ, Renan Felipe Correia ${ }^{2}$, Alex Natalino Ribeiro², Samuel Ribeiro dos Santos Neto², \\ Ivaldo Brandão Vieira ${ }^{1,3}$, José Irineu Gorla ${ }^{1}$
}

\begin{abstract}
Background: In sports such as wheelchair rugby, the correct assessment and quantification of an athletes' peak of torque and fatigue index in sport-specific actions are essential in monitoring an athletes' training performance. The increase in the number of people practicing Paralympic sports has been growing exponentially, contributing to an increase of competition level and of athletes' performance. Objective: Thus, physical training for Paralympic sport must aim to maximize athletes' fitness potential by investigating and evaluating what variables are most important in determining ideal sporting performance. Methods: Six high-level wheelchair rugby athletes had their wheelchair propulsion peak of torque and fatigue index evaluated by isokinetic dynamometry in three different angular velocity and repetition schemes. Results: The athletes presented no significant statistical difference in peak of torque and fatigue index for both arms at different number of repetitions and angular velocity. Conclusions: This shows that independent of dexterity parameters or duration of stimuli, these high-level athletes display this motor skill in constant levels of performance. These results will allow coaches to set up normative parameters to test and evaluate their players' performances.
\end{abstract}

Key Words: Wheelchair Rugby, Paralympic Sports, Peak of Torque, Fatigue Index

\section{INTRODUCTION}

The increase in the number of people practicing Paralympic sports has been growing exponentially according to data from the International Paralympic Committee ${ }^{(1)}$. This directly contributes to an increase of competition level and of athletes' performance. Since the main objective of physical training in Paralympic sport is to maximize athletes' fitness potential ${ }^{(2,3)}$, the need arises to investigate and evaluate what variables are most important in determining ideal sporting performance for these modalities ${ }^{(4,5)}$.

It is known that muscle strength is a factor of great influence on athletic performance. Explosive strength, for example, is a crucial component in sprint performance ${ }^{(6)}$. Dowson et al. ${ }^{(7)}$ supported the idea that the magnitude of force generated during dynamic muscle contraction is related to the amount of velocity an athlete can produce during a sprint. The ability to sprint intermittently is critical to many sports, and in Wheelchair Rugby this is no different ${ }^{(8)}$. Thus, the athlete who can maintain peak power for a longer time will be able to perform constant stimuli (rapid movement over and over again) without the early onset of fatigue ${ }^{(9)}$. Sprinting requires high power output associated with high speed of muscular contractions ${ }^{(10)}$. Although research has shown that in able bodied athletes leg force production capacity may be the key component of sprinting ${ }^{(8,11)}$, there is a lack of data observing this relation in wheelchair-specific movements and its relation to force.

In sports such as wheelchair rugby, the correct assessment and quantification of an athletes' peak torque and fatigue index in sport-specific actions are essential in monitoring an athletes' training performance ${ }^{(12-14)}$. Thus, this research aimed at contributing to this field of study by investigating these variables in actions related to wheelchair sprinting.

\section{METHODOLOGY}

\section{Subjects}

Six high level athletes comprised the subjects of this study. They presented a mean age of $30.35 \pm 6.62$ years, sport practice time of $38.33 \pm 17.77$ months, and $7.22 \pm 2.43$ years since the original onset of their spinal cord injury. All athletes are formerly classified by the International Wheelchair Rugby Federation as quadriplegic ${ }^{(15,16)}$. This study was approved by the Committee of Ethics in Research with Human Beings of the Faculty of Medical Sciences of University of Campinas, under

\footnotetext{
Corresponding Author: Renan Felipe Correia. Address: Av. Érico Veríssimo, 701 - Barão Geraldo, Campinas - SP, 13083-851. E-mail: renanfcorreia@gmail.com Telephone Number: 5519993132881

2 MARGEM Laboratory. College of Physical Education at the University of Campinas (UNICAMP), Campinas (SP), Brazil.
}

Full list of author information is available at the end of the article.

Financial Support: None

Submission date 24 January 2018; Acceptance date 04 June 2018; Publication date 19 September 2018 
protocol No. 405/2009. It followed guidelines and norms that regulate research with human beings according to Brazilian Law 196/96. All subjects signed consent forms authorizing the publication of the data found in this study

\section{Evaluation Protocol}

Isokinetic evaluation has been used in the last three decades as a preferred method in determining the functional pattern of muscle strength and balance ${ }^{(17)}$. With this methodology, it is possible to quantify torque and work values of muscle groups, as well as the agonist/antagonist performance ratio of such groups ${ }^{(18)}$. To better evaluate peak torque and fatigue index in actions related to wheelchair rugby, an isokinetic evaluation was applied using a protocol adapted for upper limbs, using a Biodex System 4 dynamometer (Biodex Medical Systems, New York, USA) in two speeds: $210 \% / \mathrm{sec}$. and $300 \% \mathrm{sec}$ as described below.

The positioning of the subject and the alignment of the joints were performed according to their positioning on their competition wheelchairs ${ }^{(19)}$. The seat was maintained at $0^{\circ}$ of rotation and $85^{\circ}$ of inclination and the dynamometer at $0^{\circ}$ of rotation and $15^{\circ}$ of inclination, as to simulate the camber angle of a wheelchair's wheel. After the subjects were comfortably seated, fixations bands were placed at trunk, hip, and thigh levels in order to stabilize these body segments and restrict as much accessory movements ${ }^{(20)}$. The dynamometer's rotation axis was aligned with the head of the subject's femur, and a wheel input adaptor was used to simulate a wheelchair's wheel. Then, the following protocol followed:

- A warm up series of five repetitions at $210 \% / \mathrm{sec}$. of concentric movements simulating the propulsion movement of a wheelchair for each arm, as shown in figures 1 and 2 .

- One minute of rest,

- A series of five repetitions at $210 \%$ sec for each arm

- Three minutes rest

- A series of 15 repetitions at 300\%/sec for each arm

- Three minutes rest

- A series of 30 repetitions at $300^{\circ}$ / sec for each arm

To our knowledge and research, no studies have been published with information on how many propulsions a wheelchair rugby player performs in different game actions ${ }^{(21)}$. Thus, we chose the number of repetitions for our study based on personal experience and many years of coaching the modality, in an attempt to closely approach the reality of the game itself.

\section{Statistics Analysis}

R-plus ${ }^{\circledR}$ 2.14 Software was used for graphical analysis of the results (quarters, symmetry and curtosis) and ANOVA testing between different means. A significance level of $5 \%$ ( $p=<0.05)$ was adopted.

\section{RESULTS}

In this study, were involved as subjects high-level athletes with spinal cord injuries. Although some published research exists with this specific population, no reference values for the variables studied exists in the scientific literature. For this reason, we will present our data in its totality (Table 1 and Figures 3-6), without assessing qualitative judgement on the athletes' performance. In addition, despite the references values present in the Biodex Isokinetic machine

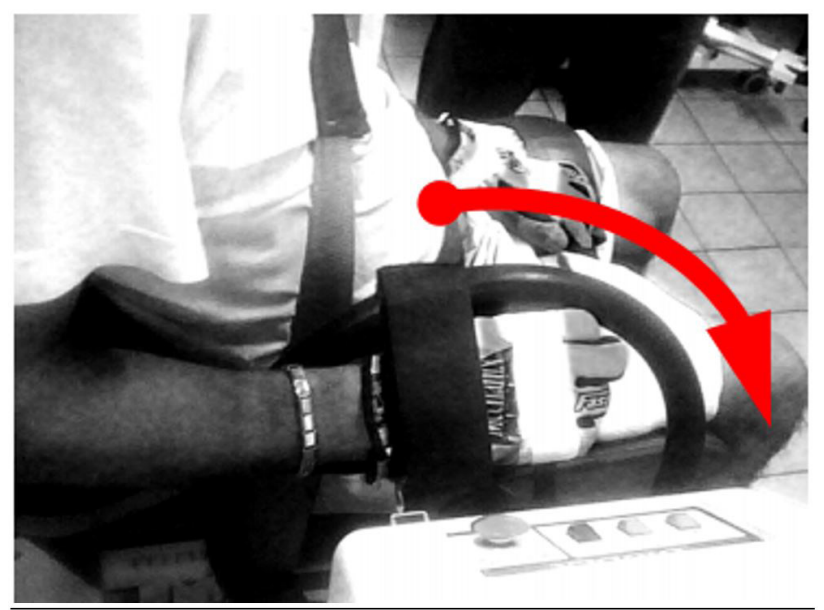

Figure 1 - Initial phase of simulated propulsion movement. Source: Authors' Personal Archive

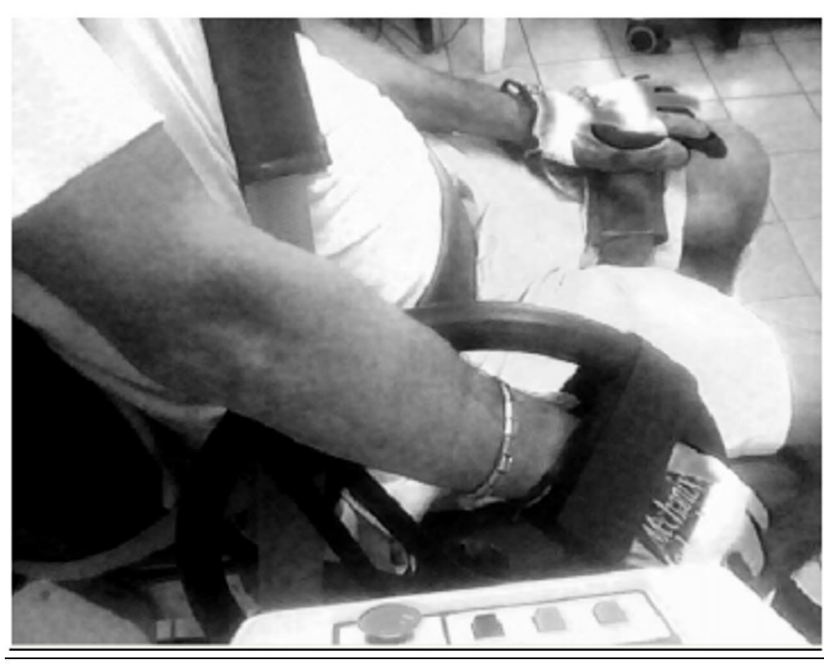

Figure 2 - Final phase of simulated propulsion movement. Source: Authors' Personal Archive 
Table 1 - Peak torque and fatigue index mean values for the right and left arms

\begin{tabular}{lcccc}
\hline \multicolumn{1}{c}{ Series } & PTRA (N/m) & PTLA (N/m) & FIRA (\%) & FILA (\%) \\
\hline $\begin{array}{l}5 \text { repetitions } \\
\text { @ 210/sec }\end{array}$ & $45.53 \pm 19.21$ & $39.85 \pm 18.74$ & $10.23 \pm 19.99$ & $31.56 \pm 5.78$ \\
15 repetitions & $44.15 \pm 18.08$ & $35.83 \pm 15.71$ & $17.43 \pm 11.54$ & $27.66 \pm 14.63$ \\
@ 300\%/sec & & & & \\
30 repetitoins & $43.68 \pm 17.74$ & $34.2 \pm 15.87$ & $31.26 \pm 8.71$ & $29.28 \pm 4.54$ \\
@ 300\%/sec & & & & \\
\hline
\end{tabular}

Note: PTRA = Peak of Torque of the Right Arm, PTLA = Peak of Torque of the Left Arm, FIRA = Fatigue Index of the Right Arm, FILA = Fatigue Index of the Left Arm; N/m= Newton por metro.

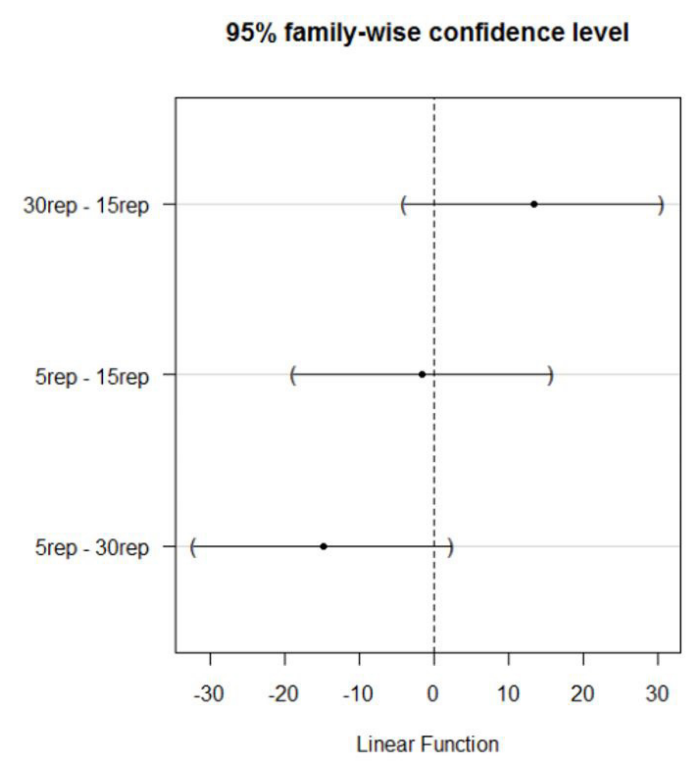

Figure 3 - Comparison between groups for the fatigue index of the right arm variable

$95 \%$ family-wise confidence level

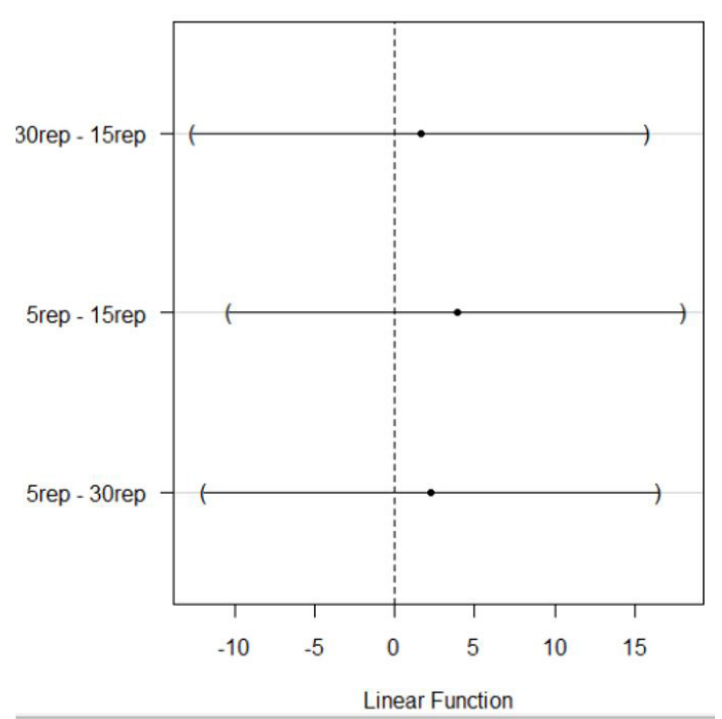

Figure 4 - Comparison between groups for the fatigue index of the left arm variable
$95 \%$ family-wise confidence level

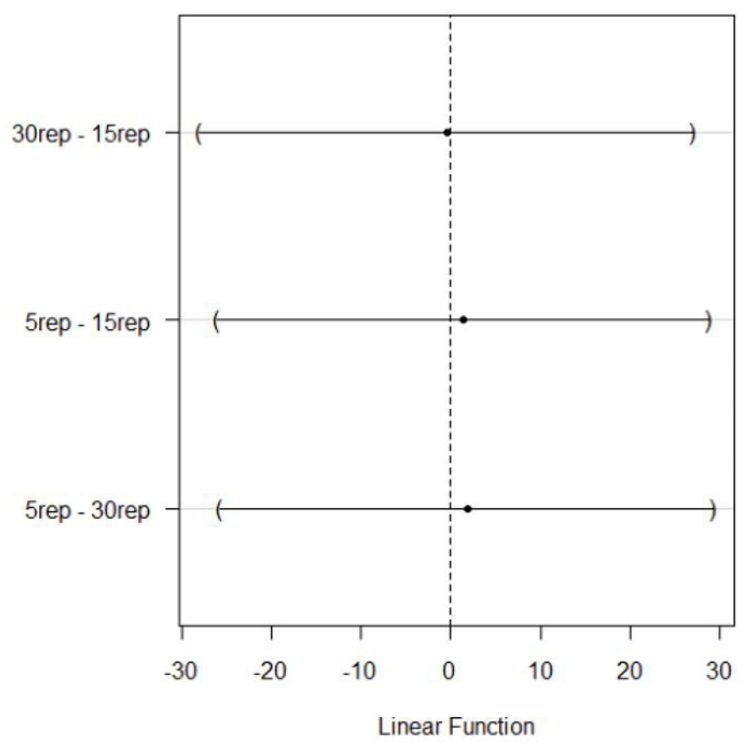

Figure 5 - Comparison between groups for the peak of torque for the right arm variable

$95 \%$ family-wise confidence level

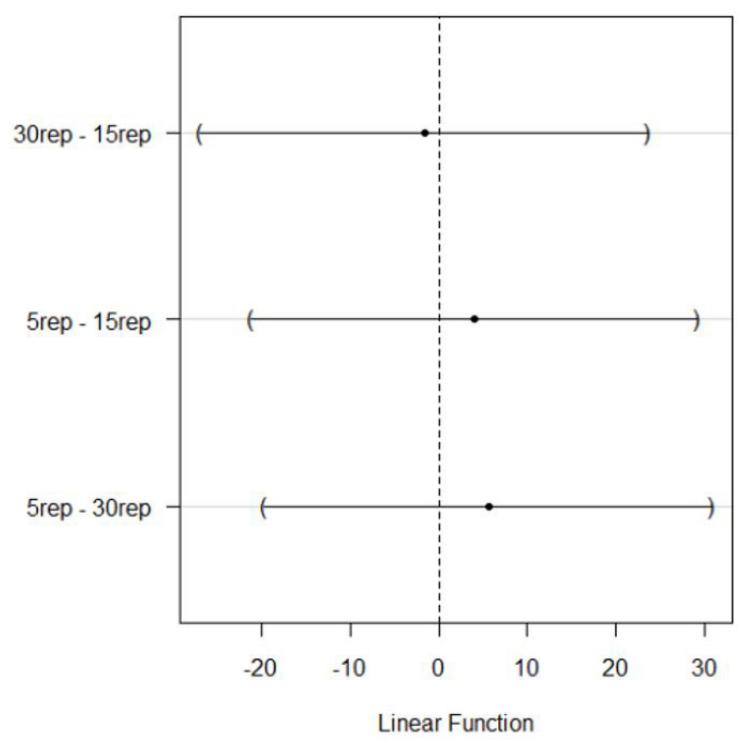

Figure 6 - Comparison between groups for the peak of torque for the left arm variable

(Biodex Medical Systems, New York, USA) Manual for high and low peak of torque and fatigue index, they were not taken into account as they were standardized for a different public.

\section{DISCUSSION AND FINAL CONSIDERATIONS}

As can be seen in the data above, results show that the athletes presented no significant statistical difference in peak of torque and fatigue index for both arms at different 
number of repetitions and angular velocity. This shows that independent of dexterity parameters or duration of stimuli, these high-level athletes display this motor skill in constant levels of performance. We expected these results for the fatigue index parameter, as wheelchair propulsion is a concentric-only action similar to bike pedaling, thus naturally allowing the athlete to perform less muscular work. However, the results for the peak of torque were surprising, as the protocol varied greatly between the stimuli (5-30), which ranged from purely anaerobic to aerobic actions. This may be a testament of these athletes' adaptation and conditioning level. A practical application of this finding is that wheelchair rugby coaches can monitor these parameters in their players in order to monitor performance and the onset of fatigue, which in turn will allow them to organize better training plans for their athletes, as well as prevent injuries ${ }^{(22,23)}$. With this, we hope that our findings further captivate the interest of Paralympic sports researchers, which in turn will increase the amount and quality of scientific information on Paralympic sports available to the academic and general public.

\section{AUTHOR'S CONTRIBUTION}

FM and JIG drafted the original research design; FM carried out the acquisition of data; FM, RFC, ANR, and SRSN analysied the data; FM, RFC, ANR, SRSN drafted and revised the original draft; JIG and IBV supervised all steps of the research; All authors approved of the version to be published.

\section{CONFLICTS OF INTEREST}

Nothing to declare.

\section{AUTHOR DETAILS}

1 Physical Evaluation Laboratory in Exercise and Adapted Sports. College of Physical Education at the University of Campinas (UNICAMP), Campinas (SP), Brazil.

${ }^{3}$ Vice-President of the Brazilian Paralympic Committee

\section{REFERENCES}

1. IPC. 2017 [cited 2017 05/05/2017]. Available from: www; paralympic.org/ IPC.

2. Barfield JP, Malone LA, Arbo C, Jung AP. Exercise intensity during wheelchair rugby training. Journal of sports sciences. 2010;28(4):389-98.

3. Bernardi M, Guerra E, Di Giacinto B, Di Cesare A, Castellano V, Bhambhani Y. Field evaluation of paralympic athletes in selected sports: implications for training. Medicine and science in sports and exercise. 2010;42(6):12008.

4. Paulson TA, Mason B, Rhodes J, Goosey-Tolfrey VL. Individualized Internal and External Training Load Relationships in Elite Wheelchair Rugby Players. Frontiers in physiology. 2015;6:388.

5. Gorla JI, Costa e Silva Ade A, Borges M, Tanhoffer RA, Godoy PS, Calegari DR, et al. Impact of Wheelchair Rugby on Body Composition of Subjects With Tetraplegia: A Pilot Study. Archives of physical medicine and rehabilitation. 2016;97(1):92-6.

6. Thorland WG, Johnson GO, Cisar CJ, Housh TJ, Tharp GD. Strength and anaerobic responses of elite young female sprint and distance runners. Medicine and science in sports and exercise. 1987;19(1):56-61.
7. Dowson MN, Nevill ME, Lakomy HK, Nevill AM, Hazeldine RJ. Modelling the relationship between isokinetic muscle strength and sprint running performance. Journal of sports sciences. 1998;16(3):257-65.

8. Baker DG, Newton RU. Comparison of lower body strength, power, acceleration, speed, agility, and sprint momentum to describe and compare playing rank among professional rugby league players. Journal of strength and conditioning research. 2008;22(1):153-8.

9. Ferriero G, Colombo R, Sartorio F, Vercelli S. Reliability of the isokinetic fatigue index. European journal of physical and rehabilitation medicine. 2011;47(2):351-2.

10. Mero A, Komi PV, Gregor RJ. Biomechanics of sprint running. A review. Sports medicine. 1992;13(6):376-92.

11. Alexander MJ. The relationship between muscle strength and sprint kinematics in elite sprinters. Canadian journal of sport sciences $=$ Journal canadien des sciences du sport. 1989;14(3):148-57.

12. Rhodes JM, Mason BS, Paulson TAW, Goosey-Tolfrey VL. Altering the Speed Profiles of Wheelchair Rugby Players With Game-Simulation Drill Design. International journal of sports physiology and performance. 2018:1-7.

13. Morgulec-Adamowicz N, Kosmol A, Molik B, Yilla AB, Laskin JJ. Aerobic, anaerobic, and skill performance with regard to classification in wheelchair rugby athletes. Research quarterly for exercise and sport. 2011;82(1):61-9.

14. Rhodes JM, Mason BS, Malone LA, Goosey-Tolfrey VL. Effect of team rank and player classification on activity profiles of elite wheelchair rugby players. Journal of sports sciences. 2015;33(19):2070-8.

15. Molik B, Lubelska E, Koxmol A, Bogdan M, Yilla AB, Hyla E. An examination of the international wheelchair rugby Federation classification system utilizing parameters of offensive game efficiency. Adapted physical activity quarterly: APAQ. 2008;25(4):335-51.

16. Altmann VC, Groen BE, van Limbeek J, Vanlandewijck YC, Keijsers NL. Reliability of the revised wheelchair rugby trunk impairment classification system. Spinal cord. 2013;51(12):913-8.

17. Gentil P, Campos MH, Soares S, Costa GCT, Paoli A, Bianco A, et al. Comparison of elbow flexor isokinetic peak torque and fatigue index between men and women of different training level. European journal of translational myology. 2017;27(4):7070.

18. Kordi M, Goodall S, Barratt P, Rowley N, Leeder J, Howatson G. Relation between Peak Power Output in Sprint Cycling and Maximum Voluntary Isometric Torque Production. Journal of electromyography and kinesiology: official journal of the International Society of Electrophysiological Kinesiology. 2017;35:95-9.

19. Braganca S, Castellucci I, Gill S, Matthias P, Carvalho M, Arezes P. Insights on the apparel needs and limitations for athletes with disabilities: The design of wheelchair rugby sports-wear. Applied ergonomics. 2018;67:925.

20. Altmann VC, Groen BE, Hart AL, Vanlandewijck YC, Keijsers NLW. Classifying trunk strength impairment according to the activity limitation caused in wheelchair rugby performance. Scandinavian journal of medicine \& science in sports. 2017.

21. Haydon DS, Pinder RA, Grimshaw PN, Robertson WSP. Overground Propulsion Kinematics and Acceleration in Elite Wheelchair Rugby. International journal of sports physiology and performance. 2017:1-23.

22. Bauerfeind J, Koper M, Wieczorek J, Urbanski P, Tasiemski T. Sports Injuries in Wheelchair Rugby - A Pilot Study. Journal of human kinetics. 2015;48:123-32.

23. Barfield JP, Newsome L, John EB, Sallee D, Frames C, Soangra R, et al. A case report of shoulder fatigue imbalance in wheelchair rugby: implications to pain and injury. Spinal cord series and cases. 2016;2:16002. 